Editorial

\title{
The Thoracic and Cardiovascular Surgeon and the Pediatric Cardiologist
}

\author{
Sven Dittrich ${ }^{1, a}$ \\ ${ }^{1}$ Department of Pediatric Cardiology, Friedrich-Alexander University \\ Erlangen-Nürnberg, Erlangen, Germany \\ Thorac Cardiovasc Surg 2019;67:330.
}

The Thoracic and Cardiovascular Surgeon together with the German Society for Pediatric Cardiology and Congenital Heart Defects introduces a supplement "Pediatric Cardiology" and calls international scientists of the field for their contributions. If you are a custom reader (and a thoracic and/or cardiovascular surgeon), you will now get the chance to scroll through this Open Access electronic supplement and to inform yourself about current research in pediatric cardiology and congenital heart disease. On the other hand, your contributions to the entire scope of thoracic and cardiovascular surgical research will now more easily attain the attention of additional pediatric and congenital cardiologists, who will become new subscribers of The Thoracic and Cardiovascular Surgeon in greater numbers. We hope for an inspiring cooperation.

The idea for this project was born during the annual scientific meetings of the German Society for Thoracic and Cardiovascular Surgery and the German Society for Pediatric Cardiology and Congenital Heart Defects, which, since 2016, have been held together at the same venue with parallel and combined scientific sessions. Surgery for congenital heart defects has always been an ambitious domain of cardiac surgery and has been always closely attended and driven by pediatric cardiologists since our first common steps by Helen Taussig and Alfred Blalock in Baltimore 1944 (and their shared publication in JAMA 1945). The basis of this relationship until this day is mutual respect and acceptance, the common interest to offer the best treatment to common patients, and the willingness to learn from each other. From the beginning surgeons and pediatricians have experienced

aresident of the German Society for Pediatric Cardiology and Congenital Heart Defects

Address for correspondence

Prof. Dr. Sven Dittrich,

Department of Pediatric

Cardiology, Friedrich-Alexander

University Erlangen-Nürnberg,

Loschgestrasse 15, 91054

Erlangen, Germany

(e-mail: sven.dittrich@uk-erlangen.de). that hand-in-hand treatment and research provide the best results and both, the progress in thoracic and cardiovascular surgery as well as the development and progress of cardiologic treatment and interventions, have made treatment of congenital heart disease the success story it currently is. In the past, many surgical as well as interventional innovations have made profound scientific impact far beyond the relatively small group of patients with congenital heart disease. And the presence of our meetings shows that there is still a broad overlap of scientific needs and interests.

From the perspective of the German Society for Pediatric Cardiology and Congenital Heart Defects there is one more reason to open the journal for Pediatric Cardiology. The publication scene is rapidly changing, and in the environment of aggressive predator journals there is a lack of serious journals offering original peer-review publications in the specialist field of pediatric cardiology and congenital heart defects. The German Society of Pediatric Cardiology and Congenital Heart Defects, in cooperation with Thieme publishers, wants to close this gap, offering all scientists in the field - the younger and the experienced ones - an attractive communication platform for their valuable scientific work. All publications of the Pediatric Cardiology supplement will be Open Access publications.

Will this concept be accepted by authors and readers?

Initially, we kindly ask you to support this project with your interest and input, giving us the chance to assure you of the additional scientific value of a Pediatric Cardiology supplement for The Thoracic and Cardiovascular Surgeon. (c) 2019 Georg Thieme Verlag KG
Stuttgart $\cdot$ New York
DOI https://doi.org/ $10.1055 / \mathrm{s}-0039-1693656$. ISSN 0171-6425. 